\title{
Ising ferromagnetism and robust half-metallicity in two-dimensional honeycomb-kagome $\mathrm{Cr}_{2} \mathrm{O}_{3}$ layer
}

\author{
Arqum Hashmi $\mathbb{1}^{1,2 凶}$, Kenta Nakanishi ${ }^{3}$, Muhammad Umar Farooq ${ }^{4}$ and Tomoya Ono ${ }^{5 凶}$
}

In contrast to the current research on two-dimensional (2D) materials, which is mainly focused on graphene and transition metal dichalcogenide-like structures, studies on 2D transition metal oxides are rare. By using ab initio calculations along with Monte Carlo simulations and nonequilibrium Green's function method, we demonstrate that the transition metal oxide monolayer $\left(\mathrm{ML}^{2}\right)$ of $\mathrm{Cr}_{2} \mathrm{O}_{3}$ is an ideal candidate for next-generation spintronics applications. $2 \mathrm{D} \mathrm{Cr}_{2} \mathrm{O}_{3}$ has honeycomb-kagome lattice, where the Dirac and strongly correlated fermions coexist around the Fermi level. Furthermore, the spin exchange coupling constant shows strong ferromagnetic (FM) interaction between $\mathrm{Cr}$ atoms. $\mathrm{Cr}_{2} \mathrm{O}_{3} \mathrm{ML}$ has a robust half-metallic behavior with a large spin gap of $\sim 3.9 \mathrm{eV}$ and adequate Curie temperature. Interestingly, an intrinsic Ising FM characteristic is observed with a giant perpendicular magnetocrystalline anisotropy energy of $\sim 0.9 \mathrm{meV}$. Most remarkably, nonequilibrium Green's function calculations reveal that the $\mathrm{Cr}_{2} \mathrm{O}_{3} \mathrm{ML}$ exhibits an excellent spin filtering effect.

npj 2D Materials and Applications (2020)4:39; https://doi.org/10.1038/s41699-020-00174-0

\section{INTRODUCTION}

A tremendous amount of research attention is currently focused on nanomaterials because of their remarkable physical properties and diverse technological applications ${ }^{1}$. While there have been significant progress in the field of two-dimensional (2D) materials displaying a broad range of electronic and optical properties ${ }^{2}$, most of these materials are intrinsically nonmagnetic, thus hindering their application to spintronics ${ }^{3}$. Recent experimental discoveries of ferromagnetic (FM) 2D layers have demonstrated the cleavage of $\mathrm{Crl}_{3}$ (ref. ${ }^{4}$ ) and $\mathrm{Cr}_{2} \mathrm{Ge}_{2} \mathrm{Te}_{6}$ (ref. ${ }^{5}$ ) single crystals that possess a FM order even in the monolayer ( $\mathrm{ML})$. Moreover, numerous room-temperature FM thin films containing $3 d$ transition metals, such as $\mathrm{VSe}_{2}$ (ref. ${ }^{6}$ ) and $\delta$ - $\mathrm{FeOOH}^{7}$ have been synthesized on van der Waals substrates. These recent experimental recognitions of intrinsic magnetism in 2D materials have instigated the search for 2D magnetic materials that exhibit longrange ferromagnetism.

Spintronics is one of the most promising fields in condensed matter physics owing to the expectation of various applications of innovative technologies by utilizing the spin degree of freedom ${ }^{8}$. In the field of spintronics, half-metallic materials have many advantages because one spin channel is conductive, while the other is insulating 9 . This results in a $100 \%$ spin polarization and giant magnetoresistance, which are essential quantities for spintronics. Various materials are known to be half-metallic, but most of them are conventional bulk systems. 2D layers show many peculiar physical properties because of great modifications in their electronic structures at low dimension. Increase in the thickness as compared to ML has a significant effect on its properties, such as change in magnetic ordering, bandgap dependency, and metal-insulator transition. In addition, numerous quantum phases including the quantum spin and quantum anomalous Hall effect etc. are expected to be discovered in 2D layers and their heterostructures. Due to the high demand for next-generation nanoscale spintronic devices, it is necessary to develop 2D halfmetallic materials.

In contrast to the current research, which is mainly focused on the 2D graphene and transition metal dichalcogenide-like structures $^{10-14}$, very few atomically thin metal oxides ${ }^{15-17}$ and hydroxides ${ }^{18}$ have been synthesized to date. Experimental study of $2 \mathrm{D}$ magnetic layers shows the $\mathrm{Cr}$-based layers have FM state with large spin magnetic moment $\left(\geq 3 \mu_{B}\right)^{19}$. Moreover, experimental fabrication of the ultrathin $\mathrm{Cr}_{2} \mathrm{O}_{3}$ nanosheets by rapid thermal annealing ${ }^{20}$ that suggests the viability of synthesizing $2 \mathrm{D}$ $\mathrm{Cr}_{2} \mathrm{O}_{3}$ motivates us to explore the stability, electronic, and magnetic properties of $\mathrm{Cr}_{2} \mathrm{O}_{3} \mathrm{ML}$. Ab initio calculations demonstrate that the half-metallic $\mathrm{Cr}_{2} \mathrm{O}_{3} \mathrm{ML}$ satisfies the key factors for next-generation spintronics applications, such as large spin gap, magnetocrystalline anisotropy energy (MAE), and high Curie temperature $\left(T_{\mathrm{c}}\right)$. The exceptionally large spin gap of $\sim 3.9 \mathrm{eV}$ and its distinct honeycomb-kagome structure differentiate $\mathrm{Cr}_{2} \mathrm{O}_{3}$ $\mathrm{ML}$ from the recent theoretical prediction of $2 \mathrm{D}$ magnetic materials ${ }^{21-25} \cdot \mathrm{Cr}_{2} \mathrm{O}_{3} \mathrm{ML}$ displays not only an adequate $T_{\mathrm{c}}$ $(185 \mathrm{~K})$, but also a giant perpendicular MAE as compared to experimentally realized $2 \mathrm{D}$ layers ${ }^{4,5,26,27}$. Our results show that the $2 \mathrm{D} \mathrm{Cr}_{2} \mathrm{O}_{3} \mathrm{ML}$ exhibits an intrinsic Ising ferromagnetism and excellent spin filtering effect.

\section{RESULTS AND DISCUSSION}

Structural stability

The $\mathrm{Cr}_{2} \mathrm{O}_{3} \mathrm{ML}$ is a honeycomb-kagome lattice (as shown in Fig. $1 \mathrm{a})$, in which the $\mathrm{O}$ atoms form a kagome lattice, while the $\mathrm{Cr}$ atoms bonding with the $\mathrm{O}$ atoms form a hexagonal lattice. This honeycomb-kagome structure may lead to exotic magnetic properties different from the case of the pure kagome lattice. The stability of $\mathrm{Cr}_{2} \mathrm{O}_{3} \mathrm{ML}$ was evaluated from the formation energy

$E_{f}=E\left(\mathrm{Cr}_{2} \mathrm{O}_{3}\right)-2 E(\mathrm{Cr})-3 / 2 \mu\left(\mathrm{O}_{2}\right)$,

\footnotetext{
${ }^{1}$ Center for Computational Sciences, University of Tsukuba, Tsukuba, Ibaraki 305-8577, Japan. ${ }^{2}$ Kansai Photon Science Institute, National Institutes for Quantum and Radiological Science and Technology (QST), Kyoto 619-0215, Japan. ${ }^{3}$ Graduate School of Pure and Applied Sciences, University of Tsukuba, Tsukuba, Ibaraki 305-8571, Japan. ${ }^{4}$ Department of

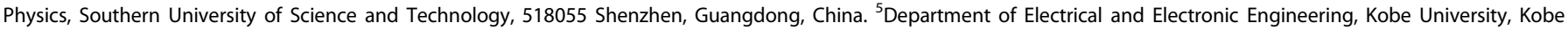
657-8501, Japan. ${ }^{凶}$ email: hashmi.arqum@qst.go.jp; t.ono@eedept.kobe-u.ac.jp
} 

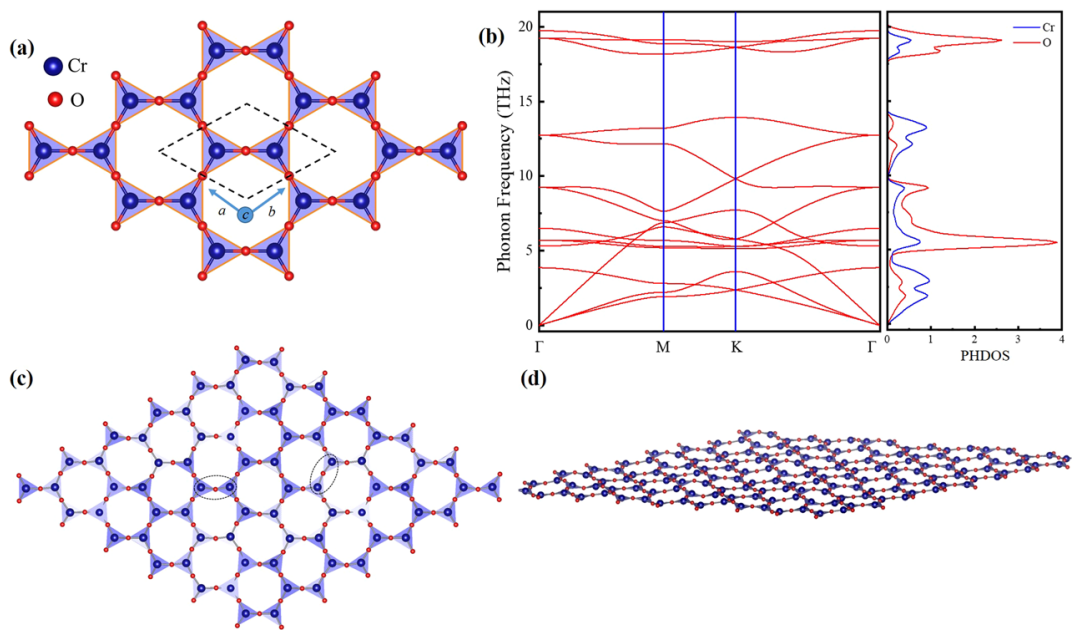

(d)

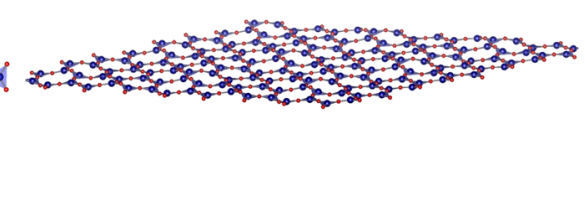

Fig. $1 \mathrm{Cr}_{2} \mathrm{O}_{3}$ monolayer and its structural stability. a Top view of $\mathrm{Cr}_{2} \mathrm{O}_{3} \mathrm{ML}$ with lattice vectors $a$ and $b$ in the $x y$ plane. Here, $a$ (c) axis is parallel to $x(z)$ axis. The rhombus shows the unit cell. $\mathbf{b}$ The calculated phonon spectrum and phonon DOS. Snapshots of $\mathbf{c}$ top and $\mathbf{d}$ side views of $\mathrm{Cr}_{2} \mathrm{O}_{3} \mathrm{ML}$ at the end of a 5-ps ab initio MD simulation at $300 \mathrm{~K}$.

where $E\left(\mathrm{Cr}_{2} \mathrm{O}_{3}\right)$ is the total energy of $\mathrm{Cr}_{2} \mathrm{O}_{3} \mathrm{ML}, E(\mathrm{Cr})$ is the energy of a $\mathrm{Cr}$ atom in the stable bulk (bcc-Cr) crystal, and $\mu\left(\mathrm{O}_{2}\right)$ is the chemical potential of oxygen gas. The formation energy of $\mathrm{Cr}_{2} \mathrm{O}_{3}$ $\mathrm{ML}$ is $-8.75 \mathrm{eV}$ atom ${ }^{-1}$, which is comparable to the graphene $\left(-8.66 \mathrm{eV} \text { atom }{ }^{-1}\right)^{28}$. The formation energy shows that the $\mathrm{Cr}_{2} \mathrm{O}_{3}$ $\mathrm{ML}$ is a strongly bonded planar network and can be prepared via well-established soft chemical routes.

The dynamical properties of the $\mathrm{Cr}_{2} \mathrm{O}_{3} \mathrm{ML}$ were evaluated by phonon dispersion calculations, as shown in Fig. 1b. Very lowfrequency phonon modes mostly originate from $\mathrm{Cr}$ atoms, while we observe $\mathrm{O}$ dominated phonon modes at $5 \mathrm{THz}$. The phonon band structure shows extremely small dispersion for these modes, thus indicating that $\mathrm{O}$ dominated frequency modes are completely localized. On the other hand, phonon band structures show that high optical modes $(\sim 18 \mathrm{THz})$ are also dominated by $\mathrm{O}$ atoms. The phonon band dispersions have no imaginary frequency modes, thus demonstrating that $\mathrm{Cr}_{2} \mathrm{O}_{3} \mathrm{ML}$ is kinetically stable. In addition, the thermal stability of the $\mathrm{Cr}_{2} \mathrm{O}_{3} \mathrm{ML}$ was assessed by performing ab initio molecular dynamics (MD) simulations at room temperature. Figure 1c, d shows snapshots of the $\mathrm{Cr}_{2} \mathrm{O}_{3} \mathrm{ML}$ at the end of $5 \mathrm{ps}$ at $300 \mathrm{~K}$. Note that the structure of the $\mathrm{Cr}_{2} \mathrm{O}_{3} \mathrm{ML}$ is intact, and all of the atomic bonds survive at room temperature. Nonetheless, the structure displays a weak out-of-plane rippling. This finding indicates that the $\mathrm{Cr}_{2} \mathrm{O}_{3} \mathrm{ML}$ can exist under realistic experimental conditions.

Magnetic properties and spin exchange coupling

The next issue to investigate is the magnetic property of the $2 \mathrm{D}$ $\mathrm{Cr}_{2} \mathrm{O}_{3} \mathrm{ML}$, with consideration of both the $\mathrm{FM}$ and antiferromagnetic (AFM) couplings between nearest-neighbor $\mathrm{Cr}$ atoms (AFM3 configuration in Fig. 2). To verify the magnetic state of $\mathrm{Cr}_{2} \mathrm{O}_{3} \mathrm{ML}$, various exchange-correlation functionals were used, including conventional local density approximation $(L D A)^{29}$, generalized gradient approximation (GGA-PBE) ${ }^{30}$, the Hubbard on-site Coulomb parameter $(U)^{31}$ of $2 \mathrm{eV}$ together with $J=0.7 \mathrm{eV}$ for $\mathrm{Cr}$ atoms to account for strong electronic correlations, and the recently developed strongly constrained and appropriately normed meta-GGA functional ${ }^{32}$. Table 1 lists the calculated energy differences between FM and AFM configurations. It is worth mentioning that all functionals show the stable FM state, as well as the same spin moment, so from this point forward, we used the GGA-PBE functional for our calculations. Figure $2 a$ presents the spin density, $\rho_{\uparrow}-\rho_{\downarrow}$, of the $\mathrm{Cr}_{2} \mathrm{O}_{3} \mathrm{ML}$, suggesting that the magnetism originates entirely from $\mathrm{FM}$ coupled $\mathrm{Cr}$ atoms, while the $\mathrm{O}$ atoms have an opposite spin with a very small magnetic moment of $0.1 \mu_{B}$.

Next, we calculated the spin exchange coupling for $\mathrm{Cr}_{2} \mathrm{O}_{3} \mathrm{ML}$. As a matter of fact, applying the isotropic Heisenberg model to a 2D FM system is against the Mermin-Wagner theorem, which claims the absence of long-range magnetic ordering at nonzero temperature ${ }^{26}$. Thus, the $2 \mathrm{D}$ Ising model is used to describe the spin coupling. $\mathrm{Cr}$ atoms are the main magnetic building block in $\mathrm{Cr}_{2} \mathrm{O}_{3} \mathrm{ML}$. Cr ions in the 2D layer have the oxidation state of +3 and are expected to give the spin $S=3 / 2$. This has been confirmed by the calculations of magnetic moment where each $\mathrm{Cr}$ carrying a spin magnetic moment of $3 \mu_{B}$. The magnetic moments localized on each $\mathrm{Cr}$ atom (local spins $S_{i, j}^{z}=3 / 2$ ) can be explained with $S=3 / 2$ spin Hamiltonian. For this purpose, we considered a $2 \times 2$ cell with four different spin configurations, as shown in Fig. 2b-e. To check the effect of supercell size on magnetic exchange coupling, we have also calculated a $4 \times 4$ cell. With the energies of four ordered spin states, three exchange constants, $J_{1}, J_{2}$, and $J_{3}$, between the first, second, and third nearest neighbors, respectively, were determined. The magnetic exchange coupling constants were calculated by

$$
\begin{aligned}
& E_{\mathrm{FM}}-E_{\mathrm{AFM} 1}=9 / 4\left(24 J_{1}+48 J_{2}+48 J_{3}\right), \\
& E_{\mathrm{FM}}-E_{\mathrm{AFM} 2}=9 / 4\left(24 J_{1}+48 J_{2}\right),
\end{aligned}
$$

and

$E_{\mathrm{FM}}-E_{\mathrm{AFM} 3}=9 / 4\left(48 J_{1}+48 J_{3}\right)$.

The calculated values of exchange constants $J_{1}, J_{2}$, and $J_{3}$ are listed in Table 2, where $J>0$ indicates FM coupling, while $J<0$ describes AFM one. All functionals display the FM interaction between first nearest neighbors and $J_{1}$ strongly dominates over others in all functionals except $\mathrm{GGA}+U$. Furthermore, all functionals show the positive $\Delta J\left(\Delta=J_{1}+J_{2}+J_{3}\right)$ value, which indicates the stable FM state of $\mathrm{Cr}_{2} \mathrm{O}_{3} \mathrm{ML}$. The values of exchange coupling in the larger cell are comparable with the smaller cell that indicates the $2 \times 2$ cell is enough to describe the magnetic exchange coupling in $2 \mathrm{D} \mathrm{Cr}_{2} \mathrm{O}_{3} \mathrm{ML}$.

Electronic properties: band structure and density of states We now discuss the electronic properties of $\mathrm{Cr}_{2} \mathrm{O}_{3} \mathrm{ML}$, which are important in terms of their application to electronic devices. GGAPBE functional was used to calculate the band structure and partial density of states (DOS). Figure $3 a$ shows that $\mathrm{Cr}_{2} \mathrm{O}_{3} \mathrm{ML}$ is a half-metallic 2D material with a large spin gap of $\sim 3.9 \mathrm{eV}$ at 


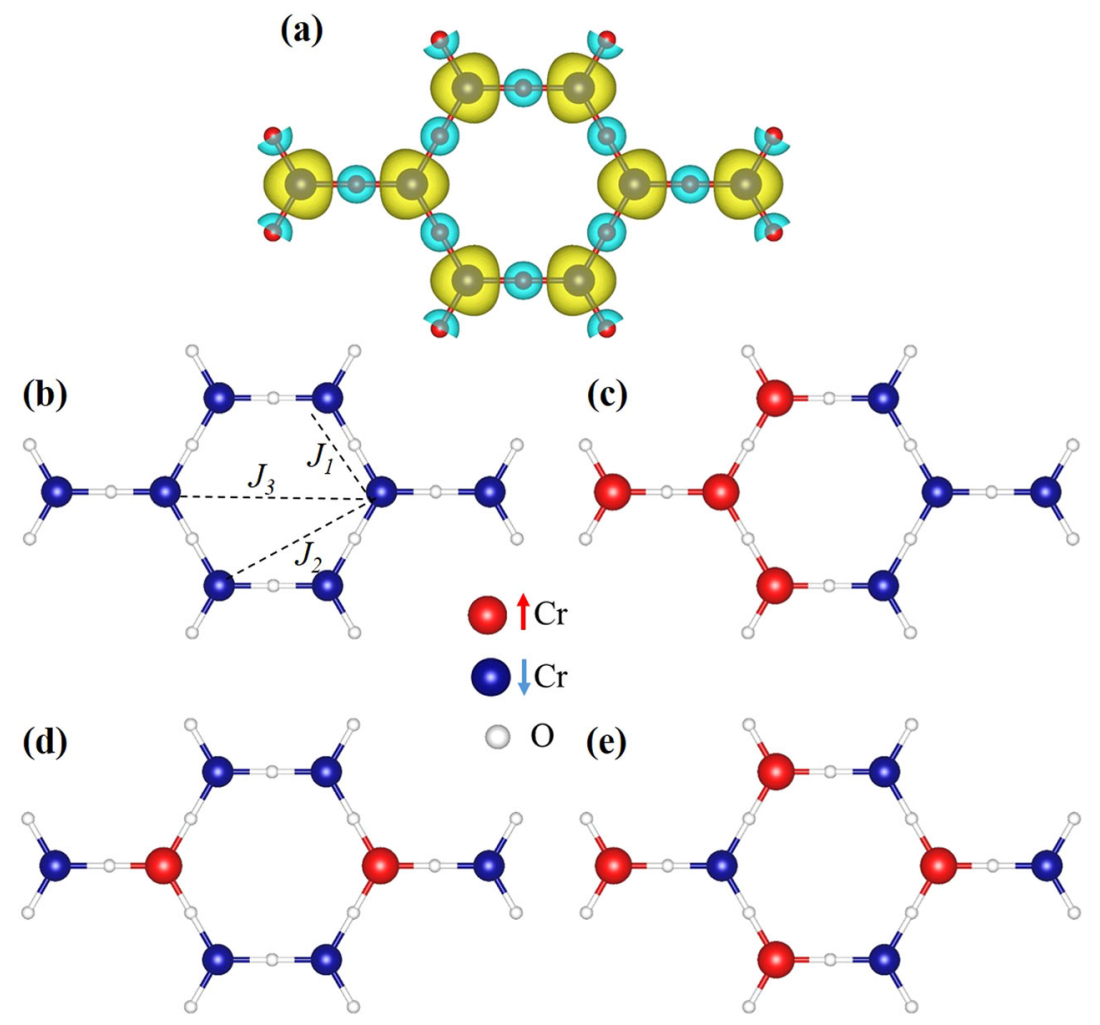

Fig. 2 Magnetization and spin exchange coupling in $\mathrm{Cr}_{2} \mathrm{O}_{3}$ monolayer. a Magnetization density for $\mathrm{Cr}_{2} \mathrm{O}_{3} \mathrm{ML}$. The gold and cyan colors have iso values of \pm 0.005 e $\AA^{-3}$. Schematic representation of four ordered spin states b FM, c AFM1, d AFM2, and e AFM3 for the determination of exchange constants. Red (blue) balls are used to highlight spins that are flipped with respect to the reference configuration. Three in-plane magnetic interactions are given as $J_{1}, J_{2}$, and $J_{3}$.

Table 1. Energy difference $\left(\Delta E=E_{\mathrm{AFM}}-E_{\mathrm{FM}}\right)$ in meV.

\begin{tabular}{lcccc}
\hline Functional & GGA & LDA & GGA $+U$ & meta-GGA \\
\hline$\Delta E$ & 263 & 203 & 109 & 200 \\
Magnetic moment/cell & 6.00 & 6.00 & 6.00 & 6.00 \\
Magnetic moment/Cr atom & 2.84 & 2.80 & 2.94 & 2.82 \\
\hline
\end{tabular}

$E_{\mathrm{FM}}$ and $E_{\mathrm{AFM}}$ denote the calculated total energies for the FM and AFM configurations, respectively. The spin magnetic moment (in $\mu_{\mathrm{B}}$ ) of unit cell and each $\mathrm{Cr}$ atom (inside Wigner-Seitz radius) of $\mathrm{Cr}_{2} \mathrm{O}_{3} \mathrm{ML}$ are presented.

Table 2. Exchange constants of $\mathrm{Cr}_{2} \mathrm{O}_{3} \mathrm{ML}$ in meV calculated using $\mathrm{FM}$ and AFM coupled phases (as shown in Fig. 2).

\begin{tabular}{lllll}
\hline Functional & GGA & LDA & GGA $+U$ & meta-GGA \\
\hline$J_{1}$ & $11.60(11.44)$ & $10.33(10.03)$ & $2.67(2.53)$ & $7.94(7.83)$ \\
$J_{2}$ & $-0.94(-1.05)$ & $-2.11(-2.13)$ & $2.40(2.32)$ & $0.53(0.49)$ \\
$J_{3}$ & $-1.83(-1.70)$ & $-2.68(-2.52)$ & $1.61(1.51)$ & $-0.34(-0.30)$ \\
\hline \multicolumn{4}{l}{ Values obtained by $4 \times 4$ cell are presented in parentheses. } \\
\hline
\end{tabular}

$\Gamma$ point. We projected the band structure for $\mathrm{Cr}_{2} \mathrm{O}_{3} \mathrm{ML}$ onto the atomic $d$ orbitals of $\mathrm{Cr}$ shown in Fig. 3b, $\mathrm{c}$ and the $p$ orbitals of $\mathrm{O}$ in Fig. 3d, e. Interestingly, typical kagome band characteristics are observed in the electronic band structure. Below the Fermi level, linearly dispersive band forms a Dirac point at the high symmetry $\mathrm{K}$ point and the corresponding two flat bands quadratically contact with the Dirac bands at the $\Gamma$ point, which are located above and below the Fermi level as marked by circles shown in
Fig. 3b. The same feature has been detected in other 2D kagome structures $^{33}$. The single-spin Dirac points below the Fermi level arise from the hybridization of the out-of-plane $\mathrm{Cr}\left(d_{x z}, d_{y z}\right)$ states with slight contributions from the $\mathrm{O}\left(d_{z}\right)$ states, while the Dirac point above the Fermi level is composed of in-plane $\mathrm{Cr}\left(d_{x y}, d_{x^{2}-y^{2}}\right)$ and $\mathrm{O}\left(p_{x y}\right)$ orbitals, as shown in Fig. $3 \mathrm{~b}, \mathrm{~d}$. The out-of-plane orbitals $d_{x z}, d_{y z}$ and $p_{z}$ form flat bands below and above the Fermi level. Flat bands are rare and emerge only in a few systems, such as twisted bilayer graphene, kagome lattices, and heavy-fermion compounds ${ }^{34}$. Since the kinetic energy of electron is quenched in the flat band, thus the out-of-plane orbitals $d_{x z}, d_{y z}$, and $p_{z}$ orbitals which form flat band have special significance. These out-of-plane orbitals indeed are responsible for the FM state in the $\mathrm{Cr}_{2} \mathrm{O}_{3}$ layer, which is explained in detail in the next section.

The magnetic exchange interaction between $\mathrm{Cr}$ atoms strongly depends on the electronic structure of $\mathrm{Cr}_{2} \mathrm{O}_{3} \mathrm{ML}$. In the case of FM configuration, $\mathrm{O}$ atoms also become spin-polarized, while in AFM case they remain nonmagnetic. Thus, $\mathrm{O}$ atom plays a vital role in the half-metallic FM state of $\mathrm{Cr}_{2} \mathrm{O}_{3} \mathrm{ML}$. No direct exchange coupling is observed between $\mathrm{O}$ and $\mathrm{Cr}$ states in AFM case and spin-polarized $d$-states are turned out to be more localized. On the other hand in $\mathrm{FM}$ configuration, we find that $\mathrm{O}(p)$ and $\mathrm{Cr}(d)$ orbitals hybridize a flat band just above the Fermi level, and this flat band degenerates with the conduction states formed by $\mathrm{Cr}$ $\left(d_{x z}, d_{y z}\right)$ orbitals at $\Gamma$ point (see Fig. 3$)$. Hence it provides an extra channel for indirect coupling between two neighboring $\mathrm{Cr}$ atoms through $\mathrm{O}$ atom, which is absent in AFM case. This conduction electron mediates indirect spin-spin coupling between $\mathrm{Cr}$ atoms through $\mathrm{O}$ atom, which is responsible for the $\mathrm{FM}$ state between $\mathrm{Cr}$ atoms in $\mathrm{Cr}_{2} \mathrm{O}_{3} \mathrm{ML}$. Figure $3 \mathrm{f}$ displays the partial DOS of the $\mathrm{Cr}_{2} \mathrm{O}_{3}$ $\mathrm{ML}$. The major contribution to the magnetic moment originates from the out-of-plane orbitals $|m|=0\left(d_{z^{2}}\right)$ and $|m|= \pm 1\left(d_{x z}, d_{y z}\right)$. 

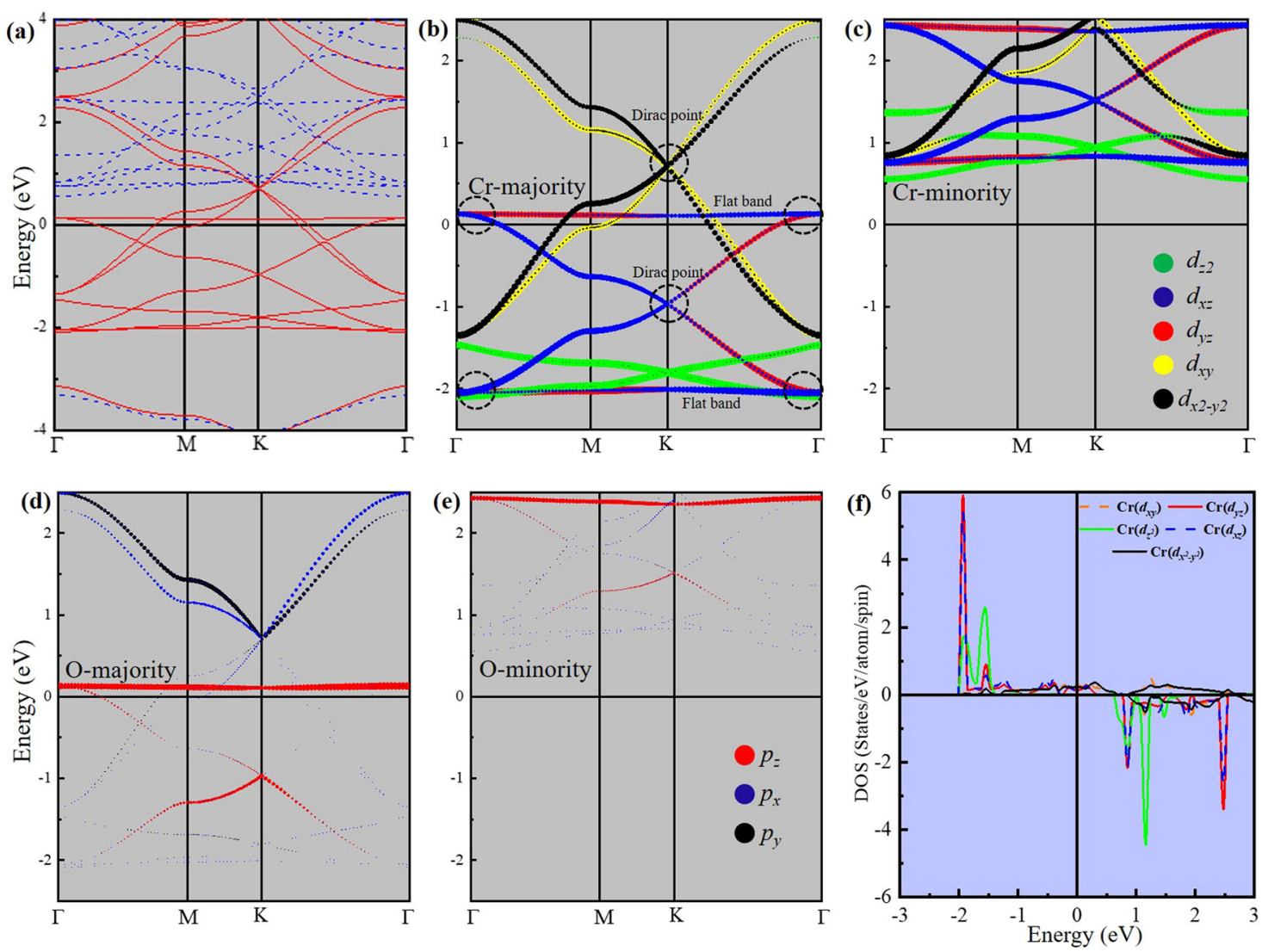

Fig. 3 Projected band structure and DOS. a Spin-resolved band structure of the $\mathrm{Cr}_{2} \mathrm{O}_{3} \mathrm{ML}$ using GGA-PBE as exchange-correlation functional. Contribution of atom-resolved band structure, $\mathrm{Cr}(d)$-resolved $\mathbf{b}$ majority spin, and $\mathbf{c}$ minority spin, while $\mathrm{O}(p)$-resolved $\mathbf{d}$ majority spin and e minority spin. The color intensity denotes the amplitude of the atom and orbital-resolved character. $\mathbf{f} \mathrm{Cr}(d)$-resolved spin-polarized DOS for $\mathrm{Cr}$ atom in $\mathrm{Cr}_{2} \mathrm{O}_{3} \mathrm{ML}$.

Magnetic anisotropy energy and curie temperature

As described before, the Mermin-Wagner theorem prohibits FM order at finite temperatures in 2D materials with continuous spin symmetries due to divergent contributions from gapless spin waves. In order to lift the restriction of continuous spin symmetry, some amount of MAE is required. Figure 4a shows the angular dependence of the MAE at the spin axis $\hat{s}(\phi, \theta)$, where $\phi$ is the polar angle which is rotated through the plane of the $a$ and $c$ axis of $\mathrm{Cr}_{2} \mathrm{O}_{3} \mathrm{ML}$, and $\theta$ is the azimuthal angle. The MAE is defined as the difference in energy between the system with a given $\hat{s}(\phi, \theta)$ and the system with spins parallel $(P)$ to the magnetic easy axis. The easy axis is found to be in the out-of-plane direction and the energy of the system with spins oriented along this direction is set to zero. MAE reaches a maximum value of $0.9 \mathrm{meV}$ at $\phi=90^{\circ}$, corresponding to an in-plane spin orientation. The value of MAE is significantly higher than bulk transition metals, such as $\mathrm{Fe}, \mathrm{Co}, \mathrm{Ni}$ $\left(1-3 \mu \mathrm{eV} \text { atom }{ }^{-1}\right)^{35}$, and the $\mathrm{Cr}_{2} \mathrm{Ge}_{2} \mathrm{Te}_{6} \mathrm{ML}\left(0.4 \mathrm{meV} \text { cell }^{-1}\right)^{26}$, while it is comparable to the $\mathrm{Crl}_{3} \mathrm{ML}\left(1.67 \mathrm{meV} \text { cell }{ }^{-1}\right)^{27}$. Figure $4 \mathrm{~b}$ illustrates the in-plane magnetization as the spin axis rotates through $a b$ plane. MAE exhibits a strong dependence on the polar angle $\phi$ and is independent of the azimuthal angle $\theta$, which is a remarkable characteristic of stable intrinsic Ising ferromagnetism. Figure $4 c, d$ depict the orbital-resolved MAE of $\operatorname{Cr}(d)$ and $O(p)$, respectively. Spin-orbit coupling (SOC) allows to calculate the matrix elements $E_{\mathrm{SOC}}$ for the angular momentum $(I=1,2)$. Following the second-order perturbation theory, the MAE can be approximately determined by matrix elements of the angular momentum operator. The matrix element difference is taken between easy axis [001] and hard axis [100]. Positive and negative values indicate the out-of-plane and in-plane contributions in
$M A E$, respectively. For $\mathrm{Cr}$ atoms, the differences of the matrix elements of $d_{x z}-d_{z^{2}}$ between in-plane and out-of-plane magnetization show large contribution for out-of-plane magnetization, while the others show negligible. For $\mathrm{O}$ atoms, the major matrix element difference of $p_{x}-p_{y}$ shows negative value, which results in the in-plane magnetization.

For practical spintronics applications, it is necessary to explore the change in magnetism of $\mathrm{Cr}_{2} \mathrm{O}_{3} \mathrm{ML}$ with respect to temperature. $T_{c}$ is a critical point at which the FM system becomes paramagnetic, and this is an important parameter to evaluate magnetic properties. We simulated the temperaturedependent magnetization curve of $\mathrm{Cr}_{2} \mathrm{O}_{3} \mathrm{ML}$ by carrying out Monte Carlo (MC) simulations ${ }^{36}$. Our model includes the MAE term in the Hamiltonian equation and can be written as

$\hat{H}=-\sum_{i, j} \sqrt{m_{i} \widehat{m}_{j}}-k_{2} \sum_{i} m_{i}^{2}$

where $\widehat{m}_{i}$ and $\widehat{m_{j}}$ are the magnetic moments (in $\mu_{B}$ ) at sites $i$ and $j$, respectively, $k_{2}$ is the anisotropy constant (MAE per atom), $m_{i}$ is the spin lying along a single preferred axis (known as the easy axis), and $J$ is the exchange parameter. For MC simulations, a $50 \times$ 50 supercell was used to mimic the $2 \mathrm{D}$ lattice; this is found to be large enough to minimize the periodic constraints. The MC simulations used 10,000 equilibrations and averaging steps. These MC simulations allow us to calculate variations in the mean magnetization per unit cell with temperature. To validate our method, we have calculated the $T_{c}$ of $\mathrm{Crl}_{3} \mathrm{ML}$ then compared it with the experimental value and the value obtained by the spinwave theory ${ }^{37}$. Spin-wave theory gives $T_{\mathrm{c}}=33 \mathrm{~K}$, underestimating the experimental value of $45 \mathrm{~K}$ by $20 \%$. In contrast, our calculated value $46 \mathrm{~K}$ is in perfect agreement with the experimental value, 

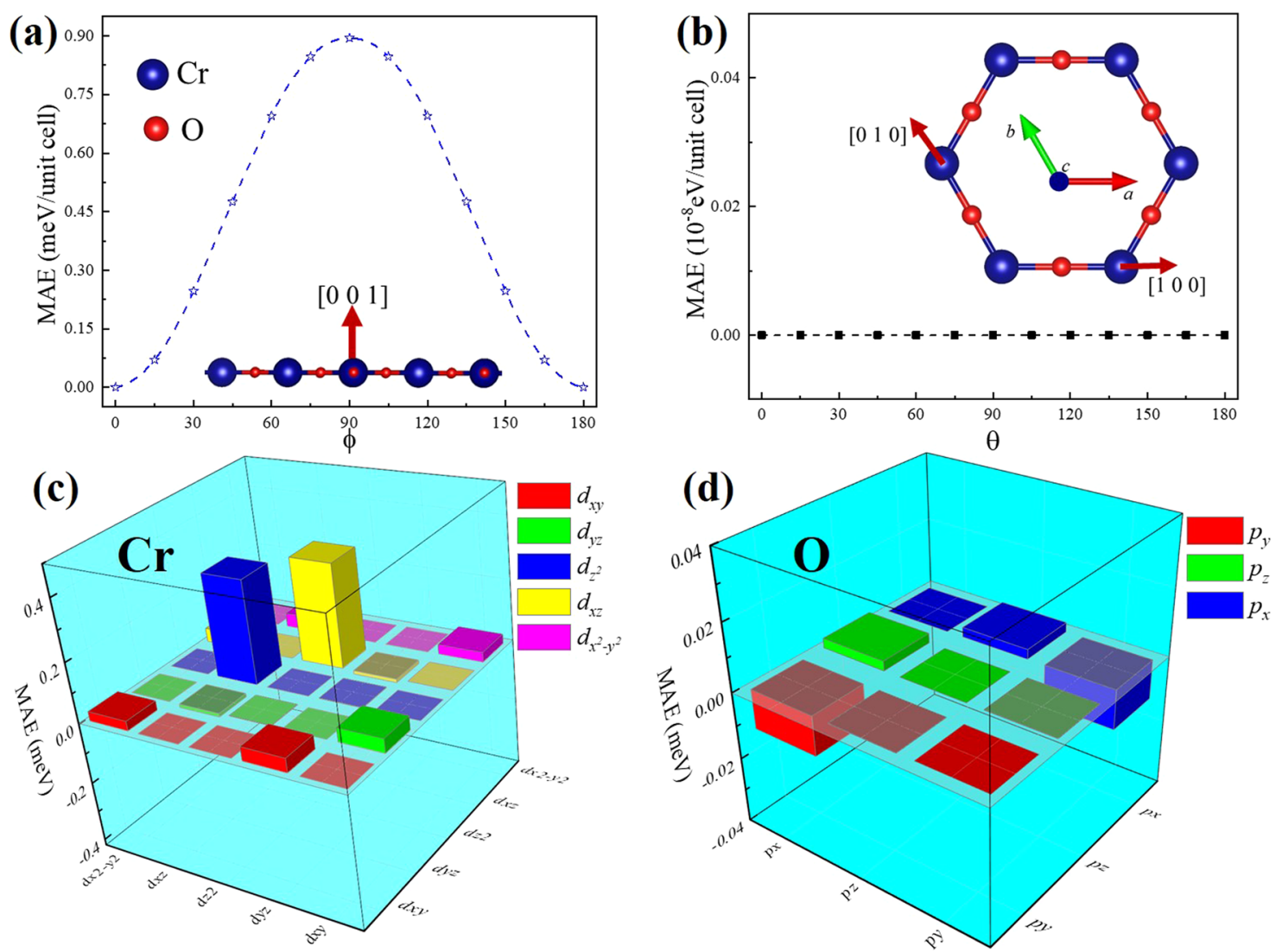

Fig. 4 Magnetocrystalline anisotropy. a Angular dependence of the $\mathrm{MAE}$ of $\mathrm{Cr}_{2} \mathrm{O}_{3}$ on polar angle $\phi$, while azimuthal angle $\theta$ is 0 . Inset shows the out-of-plane $\phi=0$ [001] direction. $\mathbf{b}$ Angular dependence of the MAE of $\mathrm{Cr}_{2} \mathrm{O}_{3}$ on $\theta$, while $\phi$ is $\pi / 2$. Inset displays the in-plane $\theta=0$ [100] and $\theta=2 \pi / 3$ [010] directions. Atom-resolved MAE of $\mathrm{Cr}_{2} \mathrm{O}_{3} \mathrm{ML}, \mathbf{c} d$-resolved $\mathrm{MAE}$ of $\mathrm{Cr}$ atom, and $\mathbf{d} p$-resolved MAE of $\mathrm{O}$ atom.

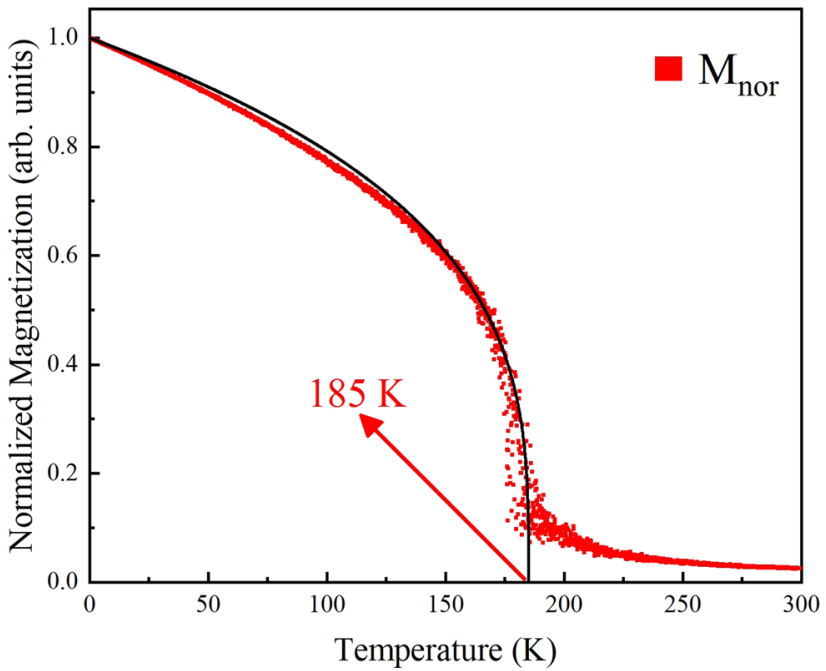

Fig. 5 Curie temperature. Temperature-dependent magnetization curve of $2 \mathrm{D} \mathrm{Cr}_{2} \mathrm{O}_{3}$ FM layer obtained by $\mathrm{MC}$ simulation.

proving the reliability of our method. The calculated mean magnetization as a function of temperature for $\mathrm{Cr}_{2} \mathrm{O}_{3} \mathrm{ML}$ is illustrated in Fig. 5. Note that the magnetization decreases to 0.77 $\mu_{B}$ at $100 \mathrm{~K}$ and the paramagnetic state is found at the temperature of $\sim 185 \mathrm{~K}$. The value of $T_{\mathrm{C}}$ is significantly higher than those of the $\mathrm{Cr}_{2} \mathrm{Ge}_{2} \mathrm{Te}_{6}(30 \mathrm{~K})^{5}, \mathrm{Crl}_{3}(45 \mathrm{~K})^{4}$, and even $\mathrm{Fe}_{3} \mathrm{GeTe}_{2}$ $(130 \mathrm{~K})^{38}$ layers.

\section{Nonequilibrium Green's function transport properties}

Lastly, to explore the spin-filtering effect at magnetic domains in half-metallic $\mathrm{Cr}_{2} \mathrm{O}_{3} \mathrm{ML}$, we calculated the spin transport properties at zero bias voltage of the device based on $\mathrm{Cr}_{2} \mathrm{O}_{3} \mathrm{ML}$ (inset in Fig. $6 \mathrm{~d})$. The corresponding position-dependent distributions of DOS for the $\mathrm{Cr}_{2} \mathrm{O}_{3}$ electrode are shown in Fig. $6 \mathrm{a}$, b. It is clear from Fig. $6 a$ that the majority spin dominates the whole energy range. On the other hand, the minority spin is concentrated away from the Fermi level in Fig. 6b. We compared the transport properties between the $P$ and antiparallel (AP) magnetization configurations, where the spin orientations of $\mathrm{Cr}$ atoms in the left and right electrodes are the same and inverse, respectively. Spin-dependent conductance for $\mathrm{P}$ and AP configurations at zero bias can be seen in Fig. $6 c$, d. P configuration of the two-probe systems (as shown in Fig. $6 \mathrm{c}$ ) is periodic with translational symmetry and the conductance for each spin takes a step form given by the number of transport channels times the conductance quantum $G_{0}=e^{2} / h$. The spin-up current is surpassed while the spin-down current is completely inhibited. In the AP configuration, as shown in Fig. 6d, the conductance spectra show no transport channel over a wide range of energy, and extremely small conductance is found away from the Fermi level in both spin-up and spin-down channels due to the similarity of the energy bands of opposite spins. Thus, both the spin-up and spin-down conductances in the AP configuration are ignorable compared to the spin-down conductance in the $P$ configuration. Our findings demonstrate that the perfect spin filtering can be achieved in a device based on $\mathrm{Cr}_{2} \mathrm{O}_{3} \mathrm{ML}$ in a wide energy range around the Fermi level.

Motivated by the experimental observation of 2D Ising FM in $M L s$, we used first-principle calculations to systemically investigate the electronic and magnetic properties of $\mathrm{Cr}_{2} \mathrm{O}_{3} \mathrm{ML}$. We 

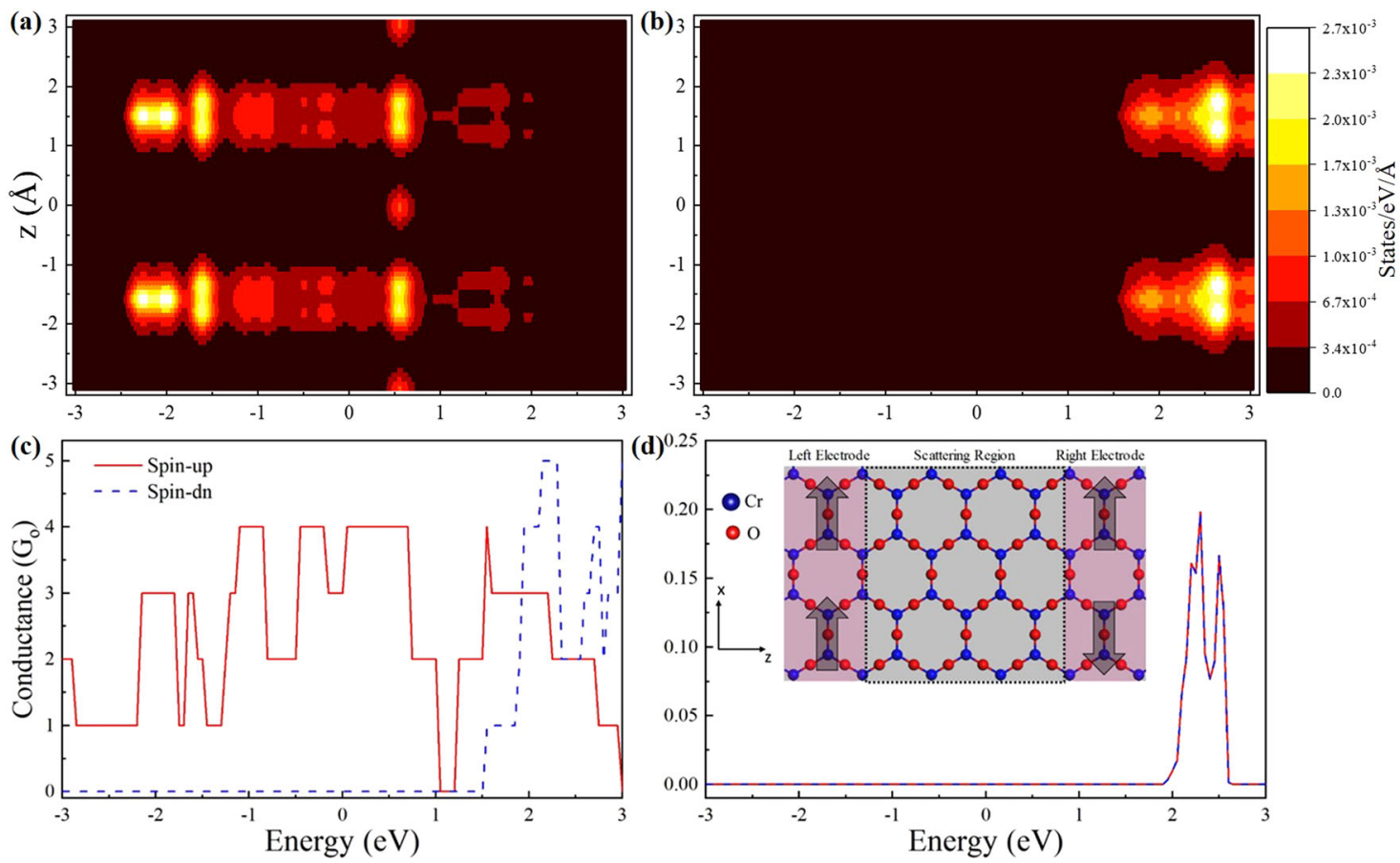

Fig. 6 Transport properties of $\mathrm{Cr}_{2} \mathrm{O}_{3}$ monolayer. Position-dependent DOS in real space for $\mathrm{Cr}_{2} \mathrm{O}_{3}$ electrode: a majority spin and $\mathbf{b}$ minority spin. The conductance of $\mathrm{Cr}_{2} \mathrm{O}_{3} \mathrm{ML}$-based device in $\mathbf{c} \mathrm{P}$ and $\mathbf{d} \mathrm{AP}$ electrode configurations. Solid and dashed curves represent spin-up and spin-down conductance, respectively. The inset shows the device model for transport calculations.

demonstrated that the honeycomb-kagome lattice of $\mathrm{Cr}_{2} \mathrm{O}_{3} \mathrm{ML}$ is thermodynamically stable at room temperature. $\mathrm{Cr}_{2} \mathrm{O}_{3} \mathrm{ML}$ has a honeycomb-kagome structure characterized by a kagome band with an unusually large gap in one spin channel. MAE exhibits a strong dependence on the polar angle $\phi$ and is independent of the azimuthal angle $\theta$, which is the characteristic of a stable intrinsic Ising ferromagnetism. Calculations of the magnetic anisotropy revealed a giant MAE of $\sim 0.9 \mathrm{meV}$. The MC simulations demonstrated that the $T_{\mathrm{c}}$ of the $\mathrm{Cr}_{2} \mathrm{O}_{3} \mathrm{ML}$ is estimated to be $185 \mathrm{~K}$. We also investigated the spin transport properties by using the nonequilibrium Green's function method, and found that the $\mathrm{Cr}_{2} \mathrm{O}_{3} \mathrm{ML}$ exhibits an excellent spin filtering. We anticipate that our work will stimulate experimental studies to validate and extend our findings.

\section{METHODS}

First-principles calculations details

The structural, electronic, and magnetic properties were calculated by using the Vienna ab initio simulation package (VASP) ${ }^{39,40}$. The interaction between valence electrons and ionic cores was described within the framework of the projector augmented wave method ${ }^{41}$. The energy cutoff for the plane wave basis expansion was set to $500 \mathrm{eV}$. The ground state optimized lattice constants of the $\mathrm{Cr}_{2} \mathrm{O}_{3} \mathrm{ML}$ were $a=b=6.175 \AA$. Self-consistent calculations were carried out with an $11 \times 11 \times 1 \mathrm{k}$-mesh and a vacuum distance of $15 \AA$ was utilized in the direction normal to the layer. The convergence criterion of energy was set to $0.01 \mathrm{meV}$ for unit cell and the atomic positions were fully relaxed until the force on each atom was smaller than $0.001 \mathrm{eV} \AA^{-1}$.

\section{Phonon and ab initio molecular dynamics}

To investigate the dynamical stability, we also calculated the phonon dispersion curve by using PHONOPY code ${ }^{42}$. Force criterion for the ionic step was set to $10^{-8} \mathrm{eV} \AA^{-1}$ for the phonon calculations. Force constant matrices used in the phonon calculations were determined by density functional perturbation theory using the VASP code with a supercell method featuring a $3 \times 3 \times 1$ supercell and $3 \times 3 \times 1 k$-points. Ab initio MD

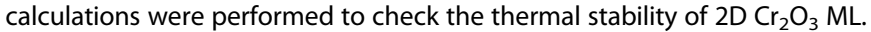
We carried out the MD simulations of $\mathrm{Cr}_{2} \mathrm{O}_{3} \mathrm{ML}$ at $300 \mathrm{~K}$. To explore this aspect, $3 \times 3$ supercell containing 45 atoms was used. The time step was set to $0.5 \mathrm{fs}$ and the simulation was run up to $5 \mathrm{ps}$. A Nosé-Hoover thermostat was used to control the ionic temperature ${ }^{43,44}$.

Magnetic anisotropy calculations details

MAEs were calculated by including SOC. A very strict criterion of $10^{-8} \mathrm{eV}$ atom $^{-1}$ for the total energy convergence was set and the converged MAE results were obtained at high $41 \times 41 \times 1 k$-points. The detailed information on the MAE calculations and the influence of different approximations using VASP can be seen in ref. ${ }^{45}$.

Nonequilibrium green's function method

For the transport calculations, we used the nonequilibrium Green's function method based on DFT. All the electron transport calculations were carried out using RSPACE code ${ }^{46-50}$, which is based on the real-space finite-difference method ${ }^{51,52}$. Exchange-correlation interactions were treated by LDA, and the norm-conserving pseudopotentials ${ }^{53}$ of Troullier and Martins ${ }^{54}$ were used for the core electrons with a grid spacing of $0.15 \AA$. The relative spin directions of two electrodes were changed to obtain zero bias-dependent transmission.

\section{DATA AVAILABILITY}

The data that supports the findings of this study are available within the article.

\section{CODE AVAILABILITY}

The calculations were implemented using the VASP, Vampire, Phonopy, and RSPACE packages.

Received: 17 June 2020; Accepted: 17 October 2020; Published online: 09 November 2020

\section{REFERENCES}

1. Mas-Ballesté, R., Gómez-Navarro, C., Gómez-Herrero, J. \& Zamora, F. 2D materials: to graphene and beyond. Nanoscale 3, 20-30 (2011). 
2. Mak, K. F. \& Shan, J. Photonics and optoelectronics of 2D semiconductor transition metal dichalcogenides. Nat. Photonics 10, 216-226 (2016).

3. Liu, Y. et al. Van der Waals heterostructures and devices. Nat. Rev. Mater. 1, 16042 (2016).

4. Huang, B. et al. Layer-dependent ferromagnetism in a van der Waals crystal down to the monolayer limit. Nature 546, 270-273 (2017).

5. Gong, C. et al. Discovery of intrinsic ferromagnetism in two-dimensional van der Waals crystals. Nature 546, 265-269 (2017).

6. Bonilla, M. et al. Strong room-temperature ferromagnetism in $\mathrm{VSe}_{2}$ monolayers on van der Waals substrates. Nat. Nanotechnol. 13, 289 (2018).

7. Chen, P. et al. Ultrathin nanosheets of feroxyhyte: a new two-dimensional material with robust ferromagnetic behavior. Chem. Sci. 5, 2251-2255 (2014).

8. Žutić, I., Fabian, J. \& Das Sarma, S. Spintronics: fundamentals and applications. Rev. Mod. Phys. 76, 323-410 (2004).

9. de Groot, R. A., Mueller, F. M., Engen, P. Gvan \& Buschow, K. H. J. New class of materials: half-metallic ferromagnets. Phys. Rev. Lett. 50, 2024-2027 (1983).

10. Zhou, X., Hang, Y., Liu, L., Zhang, Z. \& Guo, W. A large family of synthetic twodimensional metal hydrides. J. Am. Chem. Soc. 141, 7899-7905 (2019).

11. $\mathrm{Hu}, \mathrm{Y}$. et al. High Curie temperature and carrier mobility of novel $\mathrm{Fe}, \mathrm{Co}$ and $\mathrm{Ni}$ carbide MXenes. Nanoscale 12, 11627-11637 (2020).

12. Zhang, S., Xu, R., Duan, W. \& Zou, X. Intrinsic half-metallicity in $2 D$ ternary chalcogenides with high critical temperature and controllable magnetization direction. Adv. Funct. Mater. 29, 1808380 (2019).

13. Wang, B. et al. $M n X(X=P, A s)$ monolayers: a new type of two-dimensional intrinsic room temperature ferromagnetic half-metallic material with large magnetic anisotropy. Nanoscale 11, 4204-4209 (2019).

14. Choudhuri, I., Bhauriyal, P. \& Pathak, B. Recent advances in graphene-like 2D materials for spintronics applications. Chem. Mater. 31, 8260-8285 (2019).

15. Nagel, M. et al. Ultrathin transition-metal oxide films: thickness dependence of the electronic structure and local geometry in MnO. Phys. Rev. B 75, 195426 (2007).

16. Zhao, G. et al. Synthesizing $\mathrm{MnO}_{2}$ nanosheets from graphene oxide templates for high performance pseudosupercapacitors. Chem. Sci. 3, 433-437 (2012).

17. Zavabeti, A. et al. A liquid metal reaction environment for the room-temperature synthesis of atomically thin metal oxides. Science 358, 332-335 (2017).

18. Ma, R. \& Sasaki, T. Nanosheets of oxides and hydroxides: ultimate 2D chargebearing functional crystallites. Adv. Mater. 22, 5082-5104 (2010).

19. Guo, Y. et al. Magnetic two-dimensional layered crystals meet with ferromagnetic semiconductors. InfoMat 2, 639-655 (2020).

20. Zhao, C., Zhang, H., Si, W. \& Wu, H. Mass production of two-dimensional oxides by rapid heating of hydrous chlorides. Nat. Commun. 7, 12543 (2016).

21. Zhao, P., Ma, Y., Wang, H., Huang, B. \& Dai, Y. Room-temperature quantum anomalous hall effect in single-layer $\mathrm{CrP}_{2} \mathrm{~S}_{6}$. J. Phys. Chem. $C$ 123, 14707-14711 (2019).

22. Wu, Q., Zhang, Y., Zhou, Q., Wang, J. \& Zeng, X. C. Transition-metal dihydride monolayers: a new family of two-dimensional ferromagnetic materials with intrinsic room-temperature half-metallicity. J. Phys. Chem. Lett. 9, 4260-4266 (2018).

23. Wu, Q., Xu, W. W., Lin, D., Wang, J. \& Zeng, X. C. Two-dimensional gold sulfide monolayers with direct band gap and ultrahigh electron mobility. J. Phys. Chem. Lett. 10, 3773-3778 (2019).

24. Chen, $Z$. et al. Two-dimensional intrinsic ferromagnetic half-metals: monolayers $\mathrm{Mn}_{3} \mathrm{X}_{4}(\mathrm{X}=\mathrm{Te}, \mathrm{Se}, \mathrm{S})$. J. Mater. Sci. 55, 7680-7690 (2020).

25. Lv, H., Wu, D., Li, X., Wu, X. \& Yang, J. Two-dimensional transitional metal dihydride crystals with anisotropic and spin-polarized Fermi Dirac cones. J. Mater. Chem. C 6, 11243-11247 (2018).

26. Zhuang, H. L., Xie, Y., Kent, P. R. C. \& Ganesh, P. Computational discovery of ferromagnetic semiconducting single-layer $\mathrm{CrSnTe}_{3}$. Phys. Rev. B 92, 035407 (2015).

27. Moaied, M., Lee, J. \& Hong, J. A 2D ferromagnetic semiconductor in monolayer $\mathrm{Cr}$ trihalide and its Janus structures. Phys. Chem. Chem. Phys. 20, 21755-21763 (2018).

28. Ertekin, E., Chrzan, D. C. \& Daw, M. S. Topological description of the Stone-Wales defect formation energy in carbon nanotubes and graphene. Phys. Rev. B 79, 155421 (2009).

29. Ceperley, D. M. \& Alder, B. J. Ground state of the electron gas by a stochastic method. Phys. Rev. Lett. 45, 566-569 (1980).

30. Perdew, J. P., Burke, K. \& Ernzerhof, M. Generalized gradient approximation made simple. Phys. Rev. Lett. 77, 3865-3868 (1996).

31. Dudarev, S. L., Botton, G. A., Savrasov, S. Y., Humphreys, C. J. \& Sutton, A. P. Electron-energy-loss spectra and the structural stability of nickel oxide: an LSDA +U study. Phys. Rev. B 57, 1505-1509 (1998).

32. Perdew, J. P., Ruzsinszky, A., Csonka, G. I., Constantin, L. A. \& Sun, J. Workhorse semilocal density functional for condensed matter physics and quantum chemistry. Phys. Rev. Lett. 103, 026403 (2009).
33. Zhang, L. et al. Two-dimensional honeycomb-kagome $\mathrm{Ta}_{2} \mathrm{~S}_{3}$ : a promising singlespin Dirac fermion and quantum anomalous hall insulator with half-metallic edge states. Nanoscale 11, 5666-5673 (2019).

34. Yin, J.-X. et al. Negative flat band magnetism in a spin-orbit-coupled correlated kagome magnet. Nat. Phys. 15, 443-448 (2019).

35. Halilov, S. V., Perlov, A. Y. A., Oppeneer, P. M., Yaresko, A. N. \& Antonov, V. N. Magnetocrystalline anisotropy energy in cubic $\mathrm{Fe}, \mathrm{Co}$, and $\mathrm{Ni}$ : applicability of local-spin-density theory reexamined. Phys. Rev. B 57, 9557-9560 (1998).

36. Evans, R. F. L. et al. Atomistic spin model simulations of magnetic nanomaterials. J. Phys. Condens. Matter 26, 103202 (2014).

37. Lado, J. L. \& Fernández-Rossier, J. On the origin of magnetic anisotropy in two dimensional $\mathrm{Crl}_{3}$. 2D Mater. 4, 035002 (2017).

38. Fei, Z. et al. Two-dimensional itinerant ferromagnetism in atomically thin $\mathrm{Fe}_{3} \mathrm{GeTe}_{2}$. Nat. Mater. 17, 778-782 (2018).

39. Kresse, G. \& Furthmüller, J. Efficiency of ab-initio total energy calculations for metals and semiconductors using a plane-wave basis set. Comput. Mater. Sci. 6 15-50 (1996).

40. Kresse, G. \& Furthmüller, J. Efficient iterative schemes for ab initio total-energy calculations using a plane-wave basis set. Phys. Rev. B 54, 11169-11186 (1996).

41. Blöchl, P. E. Projector augmented-wave method. Phys. Rev. B 50, 17953-17979 (1994).

42. Togo, A. \& Tanaka, I. First principles phonon calculations in materials science. Scr. Mater. 108, 1-5 (2015).

43. Nosé, S. A unified formulation of the constant temperature molecular dynamics methods. J. Chem. Phys. 81, 511-519 (1984).

44. Hoover, W. G. Canonical dynamics: equilibrium phase-space distributions. Phys. Rev. A 31, 1695-1697 (1985).

45. Błoński, P. \& Hafner, J. Density-functional theory of the magnetic anisotropy of nanostructures: an assessment of different approximations. J. Phys. Condens. Matter 21, 426001 (2009).

46. Ono, T. \& Hirose, K. Timesaving double-grid method for real-space electronicstructure calculations. Phys. Rev. Lett. 82, 5016-5019 (1999).

47. Ono, T. \& Hirose, K. Real-space electronic-structure calculations with a timesaving double-grid technique. Phys. Rev. B 72, 085115 (2005)

48. Hirose, K. Ono, T. \& Fujimoto, Y. First-Principles Calculations in Real-Space Form alism: Electronic Configurations and Transport Properties of Nanostructures (Imperial College Pr, 2005).

49. Ono, T. et al. Real-space electronic structure calculations with full-potential allelectron precision for transition metals. Phys. Rev. B 82, 205115 (2010).

50. Ono, T. \& Tsukamoto, S. Real-space method for first-principles electron transport calculations: self-energy terms of electrodes for large systems. Phys. Rev. B 93, 045421 (2016).

51. Chelikowsky, J. R., Troullier, N. \& Saad, Y. Finite-difference-pseudopotential method: electronic structure calculations without a basis. Phys. Rev. Lett. 72 1240-1243 (1994).

52. Chelikowsky, J. R., Troullier, N., Wu, K. \& Saad, Y. Higher-order finite-difference pseudopotential method: an application to diatomic molecules. Phys. Rev. B 50, 11355-11364 (1994).

53. Kobayashi, K. Norm-conserving pseudopotential database (NCPS97). Comput. Mater. Sci. 14, 72-76 (1999).

54. Troullier, N. \& Martins, J. L. Efficient pseudopotentials for plane-wave calculations. Phys. Rev. B 43, 1993-2006 (1991)

\section{ACKNOWLEDGEMENTS}

This research was partially supported by MEXT as a social and scientific priority issue (Creation of new functional devices and high-performance materials to support nextgeneration industries) to be tackled by using post-K computer, JSPS KAKENHI Grant No. 19K15381, and JSPS Core-to-Core program (Controlled Interfacing of 2D materials for Integrated Device Technology). The numerical calculations were carried out using the computer facilities of the Institute for Solid State Physics at the University of Tokyo, the Center for Computational Sciences at University of Tsukuba, and the K computer provided by the RIKEN Advanced Institute for Computational Science through the HPCI System Research project (Project ID: hp190172).

\section{AUTHOR CONTRIBUTIONS}

A.H. conceived the idea and performed DFT calculations. K.N. carried out the nonequilibrium Green's function calculations. M.U.F. described the magnetic interactions of the magnetic layer. T.O. supervised the work, verified overall results, and commented on the manuscript. All the authors contributed to writing the manuscript. 


\section{COMPETING INTERESTS}

The authors declare no competing interests.

\section{ADDITIONAL INFORMATION}

Correspondence and requests for materials should be addressed to A.H. or T.O.

Reprints and permission information is available at http://www.nature.com/ reprints

Publisher's note Springer Nature remains neutral with regard to jurisdictional claims in published maps and institutional affiliations.
Open Access This article is licensed under a Creative Commons Attribution 4.0 International License, which permits use, sharing, adaptation, distribution and reproduction in any medium or format, as long as you give appropriate credit to the original author(s) and the source, provide a link to the Creative Commons license, and indicate if changes were made. The images or other third party material in this article are included in the article's Creative Commons license, unless indicated otherwise in a credit line to the material. If material is not included in the article's Creative Commons license and your intended use is not permitted by statutory regulation or exceeds the permitted use, you will need to obtain permission directly from the copyright holder. To view a copy of this license, visit http://creativecommons. org/licenses/by/4.0/.

(c) The Author(s) 2020 